\title{
Analyse de la rentabilité économique du warrantage des produits vivriers au Bénin
}

\author{
Fortuné Tchékpo OGOUVIDE ${ }^{*}$, Léonard Cossi HINNOU² et René Nestor AHOYO-ADJOVI ${ }^{3}$ \\ ${ }^{1}$ Institut National des Recherches Agricoles du Bénin / Programme d'Amélioration de la Productivité Agricole \\ des Petits Exploitants, Bénin. \\ ${ }^{2}$ Institut National des Recherches Agricoles du Bénin / Centre de Recherches Agricoles du \\ Centre-Bénin. \\ ${ }^{3}$ Institut National des Recherches Agricoles du Bénin / Direction Scientifique, Bénin. \\ *Auteur correspondant ; E-mail : fogouvide10@gmail.com, Tél (229) 97415694.
}

\author{
Received: 20-05-2021
}

Accepted: 20-10-2021

Published: 30-10-2021

\section{RESUME}

Face aux contraintes de financement de l'agriculture, le warrantage a été introduit comme une alternative potentielle pour faciliter l'accès des agriculteurs aux crédits, aux intrants et aux marchés agricoles. En dépit de la littérature abondante sur la thématique, la rentabilité comparée des modèles de warrantage mis en œuvre n'a pas été explorée. Cet article avait analysé la rentabilité économique du warrantage au Bénin, en se basant sur deux indicateurs de rentabilité : la marge nette et le taux de rentabilité interne. Au moyen d'une technique d'échantillonnage en deux étapes, les données ont été collectées auprès de 245 producteurs répartis dans 19 villages et 8 communes impliqués dans le warrantage au Bénin, au moyen d'un questionnaire structuré. Les données ont été analysées à l'aide de statistiques descriptives et de tests de comparaison de moyennes. Les résultats ont montré que les valeurs moyennes des marges nettes d'exploitation obtenues pour le warrantageAGR (1 369324 FCFA \pm 5266815 FCFA), le warrantage- Pluriel (306 955 FCFA \pm 204094 FCFA), le warrantage- commercialisation (289 889 FCFA \pm 90117 FCFA) et le warrantage-intrant (203 971 FCFA 267599 FCFA) ne sont pas statistiquement différentes. Concernant le Taux de Rentabilité Interne (TRI), les valeurs moyennes obtenues pour le warrantage-commercialisation $(94 \% \pm 5 \%)$ et du warrantage-AGR $(93 \% \pm 5,3 \%)$ n'ont pas été statistiquement différents Cependant, les TRI du warrantage-commercialisation et du warrantage-AGR sont significativement supérieurs à ceux du warrantage-intrant $(89 \% \pm 22 \%)$ et du warrantage pluriel $(75 \% \pm 18 \%)$, au seuil de $1 \%$. La promotion du warrantage devra mettre l'accent sur les modèles basés sur la commercialisation et le développement des activités génératrices de revenus.

(C) 2021 International Formulae Group. All rights reserved.

Mots clés : Warrantage, rentabilité économique, financement agricole, agriculteurs, Bénin.

\section{Analysis of the economic profitability of "warrantage" of food crops in Benin}

\section{ABSTRACT}

Faced with the agriculture financing constraints, warrantage was introduced as a potential alternative to facilitate farmers' access to agricultural credit, inputs and markets. Despite the abundant literature on the subject, the comparative profitability of the warrantage models implemented has not been explored. This article analyzed the economic profitability of warrantage system in Benin, using two profitability indicators: the net margin and 
the internal rate of return. With a two-step sampling technique, data were collected from 245 farmers in 19 villages and eight municipalities involved in warrantage in Benin, using a structured questionnaire interview. Data were analyzed using descriptive statistics and means comparison tests. The results showed that the average values of the net operating margins obtained for warrantage-AGR (1 369324 FCFA \pm 5266815 FCFA), warrantage- Pluriel (306 955 FCFA \pm 204094 FCFA), warrantage- marketing (289 889 FCFA \pm 90117 FCFA) and warrantage-input (203 971 FCFA \pm 67599 FCFA) are not statistically different. Regarding the Internal Rate of Return (IRR), the average values obtained for warrantage-marketing $(94 \% \pm 5 \%)$ and warrantage-AGR $(93 \%$ $\pm 5.3 \%$ ) were not statistically different. However, the IRRs of warrantage-marketing and warrantage-AGR are significantly higher than those of warrantage-input $(89 \% \pm 22 \%)$ and plural warrantage $(75 \% \pm 18 \%)$, at $1 \%$ threshold. The promotion of warrantage should focus on systems based on marketing and the development of income-generating activities.

(C) 2021 International Formulae Group. All rights reserved.

Keywords: Warrantage, economic profitability, agricultural financing, farmers, Benin.

\section{INTRODUCTION}

L'accès au financement est d'une importance capitale dans l'adoption de nouvelles technologies d'intensification de la production agricole développées par la recherche pour contribuer à la sécurité alimentaire (Guirkinger et Boucher, 2008). La microfinance où l'offre de services financiers (micro-crédit) aux populations pauvres, sans ressource et exclues du système bancaire, leur permet de démarrer une petite activité génératrice de revenus. Certes, le regain d'intérêt pour les institutions de microfinance en Afrique sub-saharienne est apparu avec la consécration en 2006 de Yunus par le comité du prix Nobel (Fall et Lanha, 2013). Ce regain a été aussi favorisé par la Banque Mondiale qui a décrété l'année 2005 l'année du micro-crédit. Ainsi déjà en 2007, plus de 100 millions de familles pauvres ont reçu des microcrédits à travers le monde (Daley-Harris, 2009). Suite à ces initiatives, la multiplication des intervenants (IMF, projets/programmes et ONG) et des expériences en matière de microfinance reflètent un dynamisme soutenu qui permet de penser à priori que le financement est désormais disponible et accessible à toutes les catégories d'acteurs économiques (ruraux ou urbains). Cependant, malgré la place accordée au financement du secteur agricole par les gouvernants pour assurer la sécurité alimentaire des populations, développer les exportations et améliorer les conditions de vie en milieu rural, les résultats sont en deçà des attentes. La manifestation la plus évidente de cette situation est l'émergence des crises d'impayés dans le secteur de la microfinance depuis la fin des années 2000 (2008 en Zambie, 2007-2008 au Nicaragua, en BosnieHerzégovie, au Pakistan et au Maroc, 2010 en Inde et 2011 au Bénin), indiquant que l'utilisation de la microfinance pour résoudre les défis de développement en milieu rural n'est pas une panacée (Houédjissin et al., 2002 ; Barthélemy, 2013).

Pour continuer à répondre aux difficultés de financement de l'agriculture et réduire les impayés dans ce secteur sensible mais très risqué, les institutions de microfinance ont dû inventer des approches alternatives aux formes classiques d'offre de crédit dont la plus répandue est le système de warrantage ou crédit sur nantissement de stock. Depuis son introduction, le warrantage s'est imposé comme une alternative potentielle pour faciliter l'accès des agriculteurs à la fois aux crédits, aux intrants agricoles et aux marchés (Abdoulaye et Sanders, 2003; Tabo et al., 2008 ; Duffau et al., 2011 ; Faladé, 2011). Ce faisant, le warrantage met le producteur à l'abri du bradage des produits agricoles juste après la récolte, en lui permettant d'avoir accès aux facteurs de production (intrant, capital, ...) et de profiter du différentiel temporel des prix sur le marché. Le warrantage contribue non seulement à la sécurité alimentaire du ménage, mais aussi à l'amélioration significative du revenu des producteurs, selon la forme ou l'utilisation faite du crédit obtenu (Coulter et Mahamadou, 2009 ; Faladé, 2011). 
Compte tenu de l'importance du warrantage dans la promotion de l'agriculture en milieu rurale, les travaux scientifiques qui lui ont été consacrés se sont essentiellement focalisées sur l'évolution du prix des produits stockés, les destinations des crédits et les types d'acteurs impliqués (Othman et al., 2009), l'intérêt du warrantage pour les petits producteurs agricoles (Duffau et al., 2011), l'état des lieux du système de warrantage (Faladé, 2011), la caractérisation et l'évaluation des performances des modèles de warrantage au Bénin (Egah et al., 2014), les facteurs humains, agricoles et structurels qui déterminent l'adoption des formes de warrantage basé sur le maïs (Egah et al., 2015), l'influence du crédit-warrantage sur la croissance du mouvement coopératif (cas du Burundi) (Manirakiza et al., 2018) et l'analyse de la rentabilité du warrantage du mil et du niébé (Coulter et Mahamadou, 2009). Bien que ces études se soient intéressées à la thématique, l'analyse comparative de la rentabilité des modèles du warrantage mis en œuvre par les producteurs n'est pas encore abordée. Cet article avait pour objectif de contribuer au débat scientifique, en analysant la rentabilité économique du warrantage au Bénin.

\section{MATERIEL ET METHODES \\ Données utilisées}

Les données qui ont été utilisées proviennent d'une enquête réalisée par nousmêmes au cours de la campagne agricole 20182019 dans 19 villages, répartis dans 8 communes (Boukoumbé, Matéri, Gogounou, Kandi, N'Dali, Nikki, Djidja et Kétou) impliqués dans le warrantage au Bénin. (Tableau 1). Les zones d'étude ont été sélectionnées de façon raisonnée, avec l'appui de personnes-ressources des structures (projets, programmes, ONG, Mairies, ...) impliqués dans l'accompagnement du processus de warrantage. Le principal critère de choix est la pratique du warrantage au cours de la campagne agricole 2018-2019. Ensuite, dans chaque village, les producteurs ayant participé au warrantage au cours de la campagne agricole 2018/2019 ont été recensés, en utilisant la technique d'échantillonnage par boule de neige. Au total, 245 producteurs ont été recensés et systématiquement enquêtés.

Les données ont été collectées au moyen d'un questionnaire structuré implémenté via une plateforme ODK Kobocollect. Les données concernaient les caractéristiques démographiques (âge, sexe, situation matrimoniale, niveau d'instruction, statut social, taille du ménage, appartenance à des associations, ...) et socio-économiques (activités, expérience en agriculture, superficies, connaissance du warrantage, participation au warrantage, expérience dans le warrantage, ...) des producteurs, l'accès au crédit agricole, les structures de financement des activités dans le secteur agricole, les conditions d'accès au crédit-warrantage, les types de warrantage pratiqués, les acteurs impliqués et leur rôle, les produits warrantés, les structures des coûts engagés dans le processus de warrantage (transport, conditionnement, manutention, produits de stockage/conservation, gardiennage, suivi des produits, contribution à l'entretien du magasin, taxes et cotisations, charges liées aux AGR développées avec le crédit-warrantage, amortissement des outils/matériels utilisés, ...) la valeur monétaire des quantités stockées et déstockées, la valeur du crédit reçu; l'utilisation du crédit reçu, les taux d'intérêt payé et les frais connexes qui interviennent dans le processus d'octroi de crédit, les stratégies de remboursement des crédits, les contraintes à la mise en œuvre du warrantage ainsi que les facteurs de succès.

\section{Méthodes d'analyse des données}

L'analyse des données avait consisté à calculer pour chacun des modèles de warrantage, les indicateurs de rentabilité que sont: (i) la marge nette agricole et (ii) le taux de rentabilité interne ou Taux moyen de rémunération du capital.

\section{Marge nette (MN) agricole}

La marge nette agricole est obtenue en déduisant du produit brut en valeur, les coûts totaux ou en déduisant de la marge brute les coûts fixes (Yabi, 2009 ; Paraïso et al., 2012 ; Yabi et al., 2012). Mathématiquement, on a : 


$$
\begin{gathered}
M N=P B-(C V+C F) \text { ou } M N \\
=M B-C F(\text { en FCFA } \\
\text { /producteur }) \\
\text { Les coûts variables (CV), }
\end{gathered}
$$

correspondent à la valeur des produits stockés et les dépenses liées à l'acquisition d'intrants divers (produits de conservation, maind'œuvre salariée utilisée pour gardiennage et manutention, ficelle, sacs, dépenses de transport, ...). Les coûts fixes (CF) sont principalement les dépenses d'amortissement des investissements (les bâches, les matériels de mesure, ....). Les CF ont été déterminés en appliquant un taux d'amortissement linéaire à la valeur totale d'acquisition du matériel. Ce taux correspond à l'inverse de la durée de vie du matériel.

Si la marge nette $(\mathrm{MN})>0$, l'activité de warrantage est jugée économiquement rentable. Le produit brut $(\mathrm{PB})$ permet donc de couvrir toutes les charges de l'activité et un bénéfice est dégagé. Par contre, si MN < 0 , l'activité de warrantage n'est pas économiquement rentable et est donc soldée par une perte.

\section{Taux de rentabilité interne (TRI) ou Taux moyen de rémunération du capital}

Le taux de rentabilité interne, qui n'est rien d'autre que la productivité du capital, est déterminé par la formule qui suit :

$T R I=M N /(C T+V M O)$
CT : coûts totaux de production en FCFA; VMO : valeur de la main d'œuvre familiale en FCFA. Elle est déterminée en multipliant la quantité physique de la main d'œuvre par le coût d'opportunité de la main d'œuvre dans la zone.

Le taux de rentabilité interne (TRI), exprimé en \%, mesure la marge nette (MN) par unité de capital investi. Ce capital investi correspond aux coûts totaux de production (Yabi et al., 2012). Le TRI permet également d'apprécier la capacité d'une exploitation à rentabiliser le capital investi.

Le TRI a été comparé au taux d'intérêt i appliqué par les IMF de la zone d'étude. Ainsi, si TRI $>i$, l'activité de warrantage est économiquement rentable du point de vue de l'investissement. Le prêt fait est payé à partir de la marge nette dégagée et se justifie. Mais, si au contraire, TRI $<\mathrm{i}$, l'activité de warrantage n'est pas rentable du point de vue de l'investissement fait. Le prêt fait ne se justifie pas et le producteur aurait dû aller vendre son produit déposé en stock et épargner la recette, plutôt que d'investir dans le warrantage.

En plus des statistiques descriptives, un test de comparaison des valeurs moyennes des

\begin{tabular}{|c|c|c|c|}
\hline Départements & PDA & Communes & / Villages \\
\hline \multirow[t]{2}{*}{ Atacora- } & 3 & Matéri & Kouforpissiga, Nodi, Fékérou \\
\hline & & Boukoumbé & Koukongou, Koukoua, Koukouatougou \\
\hline \multirow[t]{2}{*}{ Alibori } & 2 & Gogounou & Wara, Ouessene, Angaradébou, Sam, Sonsoro \\
\hline & & Kandi & Téri, Wonga, Kassakou \\
\hline \multirow[t]{2}{*}{ Borgou } & 4 & N'Dali & N’Dali Centre, Bori, Ouénou \\
\hline & & Nikki & Biro, Woroumagassarou \\
\hline Zou & & Djidja & Fonkpodji, Kakateou, Monsourou \\
\hline Plateau & 6 & Kétou & Kétou-Centre \\
\hline
\end{tabular}
données a été fait, au moyen du logiciel statistique STATA Version 14.

Tableau 1 : Communes et villages d'enquête.

PDA=Pôle e Développement Agricole. 


\section{RESULTATS}

\section{Typologie des systèmes/modèles de warrantage}

Le warrantage est un mécanisme par lequel les Organisations Paysannes (OP) obtiennent des prêts auprès des institutions de microfinance (IMF) pour les distribuer à leurs membres à hauteur de $70-80 \%$ de la valeur des produits stockés, afin de prévenir d'éventuelles pertes physiques (baisse de taux d'humidité, dépréciation) ou baisses des prix des produits stockés. Le crédit est généralement en espèce. Cependant, au lieu d'être en espèce, le crédit peut aussi être offert sous-forme d'intrants agricoles, l'accès aux intrants ayant une influence positive sur l'adoption de la fumure minérale comme pratique culturale de gestion de la fertilité des sols (Yabi et al., 2016). En effet, ces produits qui doivent être facile à conserver, facile à vendre et dont les prix varient dans le temps, servent de garantie (nantissement de stock) pour le prêt et sont sécurisés dans un magasin fermé à double cadenas (un cadenas pour l'OP et un cadenas pour l'IMF). Le stock est suivi et gardé dans de bonnes conditions durant 4 à 5 mois, le temps que les prix des produits agricoles augmentent sur le marché, avant d'être vendu (Sogodogo et al., 2014). Les producteurs adhèrent au dispositif de conservation/suivi rigoureux du stock au magasin et sont prêts à en payer le prix, parce qu'ils sont conscients de l'incidence sur la qualité des produits (Ba et al., 2016). Pour un meilleur résultat du warrantage, le prêt obtenu est de préférence utilisé pour développer des activités génératrices de revenu (AGR) dont les bénéfices contribuent, en plus des recettes de la vente des produits stockés, au remboursement $\mathrm{du}$ prêt. Après le remboursement du prêt et des frais financiers, le solde dégagé est ristourné aux producteurs déposants, au prorata des quantités respectives de produits déposées.

Les 4 modèles de warrantage rencontrés sont: le warrantage-pluriel orienté vers la satisfaction des besoins en intrants et les obligations sociales des producteurs (43\% des producteurs), le warrantage-AGR orienté vers le développement d'activités génératrices de revenu (29\% des producteurs), le warrantagecommercialisation orienté vers la commercialisation/vente groupée des productions des participants $(18 \%$ des producteurs), et le warrantage-intrant orienté vers l'accès des producteurs aux intrants agricoles pour faciliter l'appropriation des innovations de gestion de la fertilité des sols (GIFS) en milieu rural (10\% des producteurs) (Figure 1, Tableau 2).

\section{Acteurs et gestions du crédit-warrantage Sources du crédit - warrantage}

Les sources de financement du système de warrantage (crédit-warrantage) sont moins diversifiées que celles du crédit dont bénéficient les exploitants agricoles. Il s'agit, dans l'ordre décroissant d'importance, respectivement de SIA N'SON Microfinance (26,50\%), ALIDE (24,90\%), BETHESDA $(22,90 \%)$, CCIF $(21,20 \%)$ et CLCAM $(18,80 \%)$ (Figure 2).

\section{Partenaires impliqués dans le warrantage}

En dehors des producteurs individuels et des institutions de microfinance (IMF), les autres principaux partenaires impliqués dans la mise en œuvre du warrantage étaient, dans l'ordre décroissant d'importance: les organisations des producteurs agricoles $(72,70 \%)$, les projets/programmes $(62,4 \%)$, les partenaires techniques et financiers $(52,2 \%)$, les autorités locales (Mairies) (46,5\%), les ONG $(39,2 \%)$, les structures d'appui-conseil agricole $(13,9 \%)$ et la recherche $(3,3 \%)$ (Figure 3).

\section{Utilisation et remboursement du crédit- warrantage}

Les différentes utilisations faites du crédit-warrantage et les stratégies de remboursement sont présentées dans le Tableau 3. Il en ressort que 55\% du créditwarrantage obtenu était orienté prioritairement vers le développement d'activités génératrices 
de revenu comme le petit commerce (28\%), le paiement de la main-d'œuvre due au titre de la campagne agricole en cours (12\%), l'achat des intrants vivriers pour la prochaine campagne agricole (9\%), l'élevage/embouche (5\%) et la transformation de produits agricoles $(1 \%)$. Par ailleurs, $45 \%$ du crédit-warrantage étaient investis dans les charges non productives ou sociales que sont: la construction de maison d'habitation (32\%), la scolarisation des enfants (5\%), la consommation du ménage (4\%), la santé (2\%) et diverses cérémonies (2\%).

$\mathrm{Au}$ moment du remboursement du crédit, les ressources utilisées provenaient généralement des recettes générées par les activités de commercialisation développée (42\%), de vente des produits stockés (33\%), d'autres activités de production agricole (20\%), d'élevage/embouche (4\%), de transformation des produits agricoles $(1 \%)$ et d'autres activités (moins de 1\%).

\section{Rentabilité du système de warrantage Structure des coûts liés au stockage durant le warrantage}

Les produits agricoles warrantés au cours de la campagne agricole 2018/2019 étaient constitués du maïs $(87 \%$ des producteurs) et du soja (19\% des producteurs enquêtés). Les éléments de coûts et de recettes directement associés au warrantage sont décrits dans le Tableau 4. Il s'agissait essentiellement : de la valeur du stock, des frais financiers (dossier, épargne, crédit, intérêt) et des frais de stockage (produits de stockage, gardiennage, suivi), des frais de transaction (transport, conditionnement, manutention, entretien du magasin), de l'amortissement des outils/matériels utilisés et des taxes et cotisations. A ces charges s'ajoutaient les charges liées aux activités génératrices de revenu (AGR) développées avec le créditwarrantage. Quant aux recettes, elles provenaient de la vente des produits agricoles stockés et des AGR développées.

Indicateurs de rentabilité économiques des systèmes de warrantage

La Marge Nette (MNR) et le Taux de Rentabilité Interne (TRI) Deux paramètres ont permis d'apprécier la rentabilité économique des modèles de warrantage pratiqués au Bénin. Les résultats obtenus pour les différents modèles de warrantage ont été présentés dans le Tableau 5. Il en ressort que les valeurs des marges nettes d'exploitation obtenues ont révélé que le warrantage-AGR a été le plus rentable (1 369324 FCFA \pm 526681 FCFA). Il a été suivi du warrantage-commercialisation (289 889 FCFA \pm 90117 FCFA) et du warrantage-Pluriel (306 955 FCFA \pm 204094 FCFA). Le warrantage-intrant a été le moins rentable (203971 FCFA \pm 67599 FCFA). L'analyse de la variance (ANOVA) a révélé que les marges nettes moyennes des différents modèles de warrantage n'étaient pas significativement différentes (Tableau 5).

Par ailleurs, les valeurs des TRI étaient toutes supérieures au taux d'intérêt d'environ 3,5\% l'an appliqué par les banques béninoises sur l'épargne bancaire. Par conséquent, l'investissement dans tous les modèles de warrantage se justifiait. Cependant, l'analyse de variance (ANOVA) a révélé que les TRI du warrantage-commercialisation

(TRI $=94 \% \pm 5 \%$ ) et $\mathrm{du}$ warrantage-AGR (TRI $=93 \% \pm 5,3 \%$ ) ne sont pas statistiquement différents. Par contre, ces modèles de warrantage ont permis de mieux rentabiliser le capital investi que le warrantage-intrant $(\mathrm{TRI}=89 \% \pm 22 \%)$ et le warrantage pluriel (TRI $=75 \% \pm 18 \%$ ). Ces deux derniers modèles de warrantage étaient significativement différents entre eux et des autres modèles de warrantage au seuil de 1\% [Chi2 (3)=51.5420, Prob $>$ Chi2 $=0,000]$ (Tableau 6). 


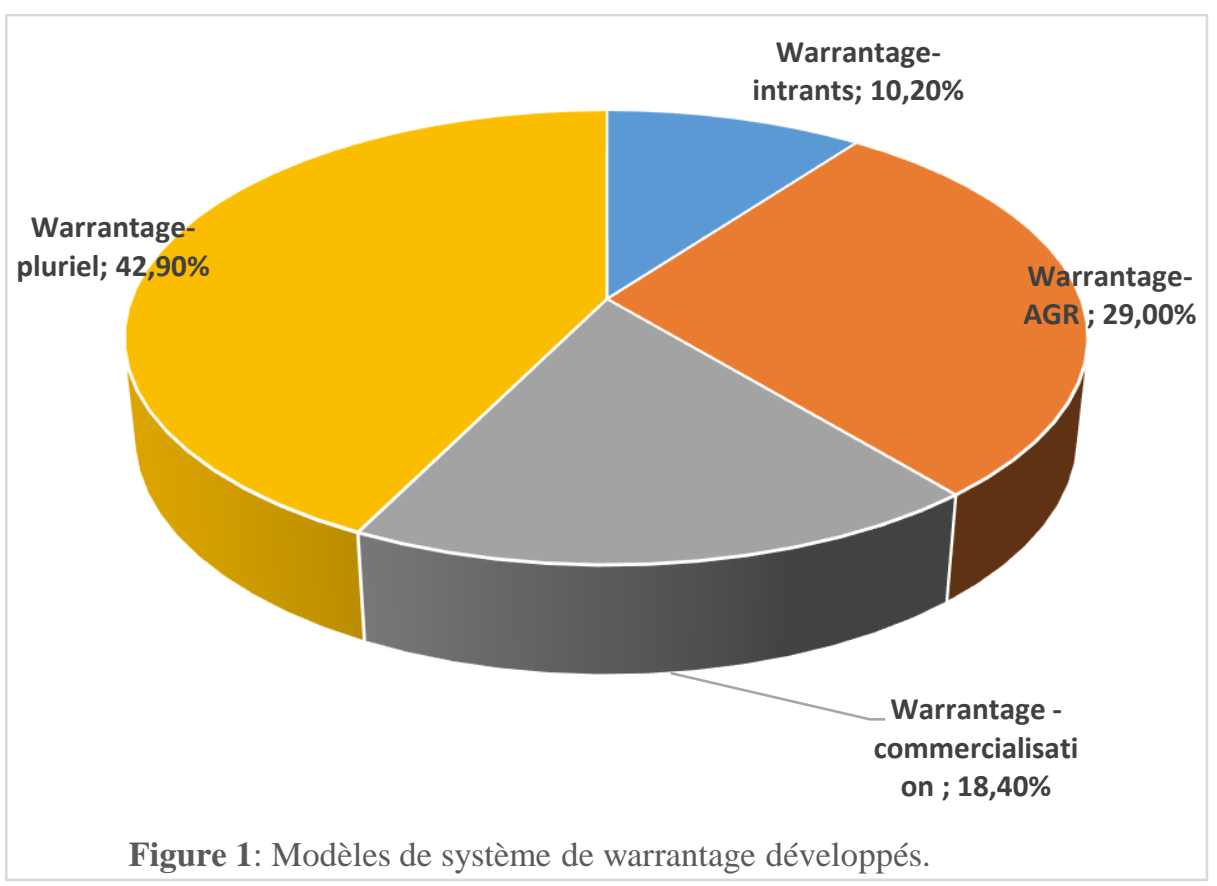

Tableau 1 : Caractérisation des modèles de warrantages pratiqués.

\begin{tabular}{lll}
\hline Modèle de warrantage & Description \\
\hline Warrantage Intrants & - & Orientation vers l'accès des producteurs aux intrants agricoles ; \\
& - & Forte implication de plusieurs types d'acteurs; \\
& - & Faible taux d'intérêt ; \\
& - & Mise en place d'un fonds de garantie pour couvrir les risques; \\
& - & Facilite surtout l'appropriation des innovations de gestion de la fertilité \\
Warrantage AGR & & des sols en milieu rural. \\
& - & Orienté vers le financement des AGR ; \\
& - & Implication d'un nombre moyen de types d'acteurs (OP, IMF, \\
& & collectivité locale, SCDA) ; \\
Warrantage & Taux d'intérêt plus élevé ; \\
Commercialisation & - & définition du montant du crédit par sac de produit ; \\
& - & Orienté vers l'écoulement des produits agricoles sur le marché ; \\
& - & Structure hiérarchique réduite (IMF, SCDA et OP) ; \\
& - & Appui technique assuré par le promoteur qui dispose des techniciens \\
& en conditionnement des produits agricoles ; \\
& - & Outil de spéculation qui permet d'améliorer les revenus moyens des \\
Warrantage pluriel & - & agriculteurs. \\
& & Combine la satisfaction des obligations sociales et des besoins en \\
& & intrants des participants ; \\
& - & Mêmes types d'acteurs que le warrantage « commercialisation ». \\
\hline
\end{tabular}

Source : Egah (2015) et Enquêtes 2019

Légende :

AGR=Activité Génératrice de Revenu

$\mathrm{IMF}=$ Institution de Microfinance

SCDA=Secteur Communal de Développement Agricole

$\mathrm{OP}=$ Organisation paysanne 


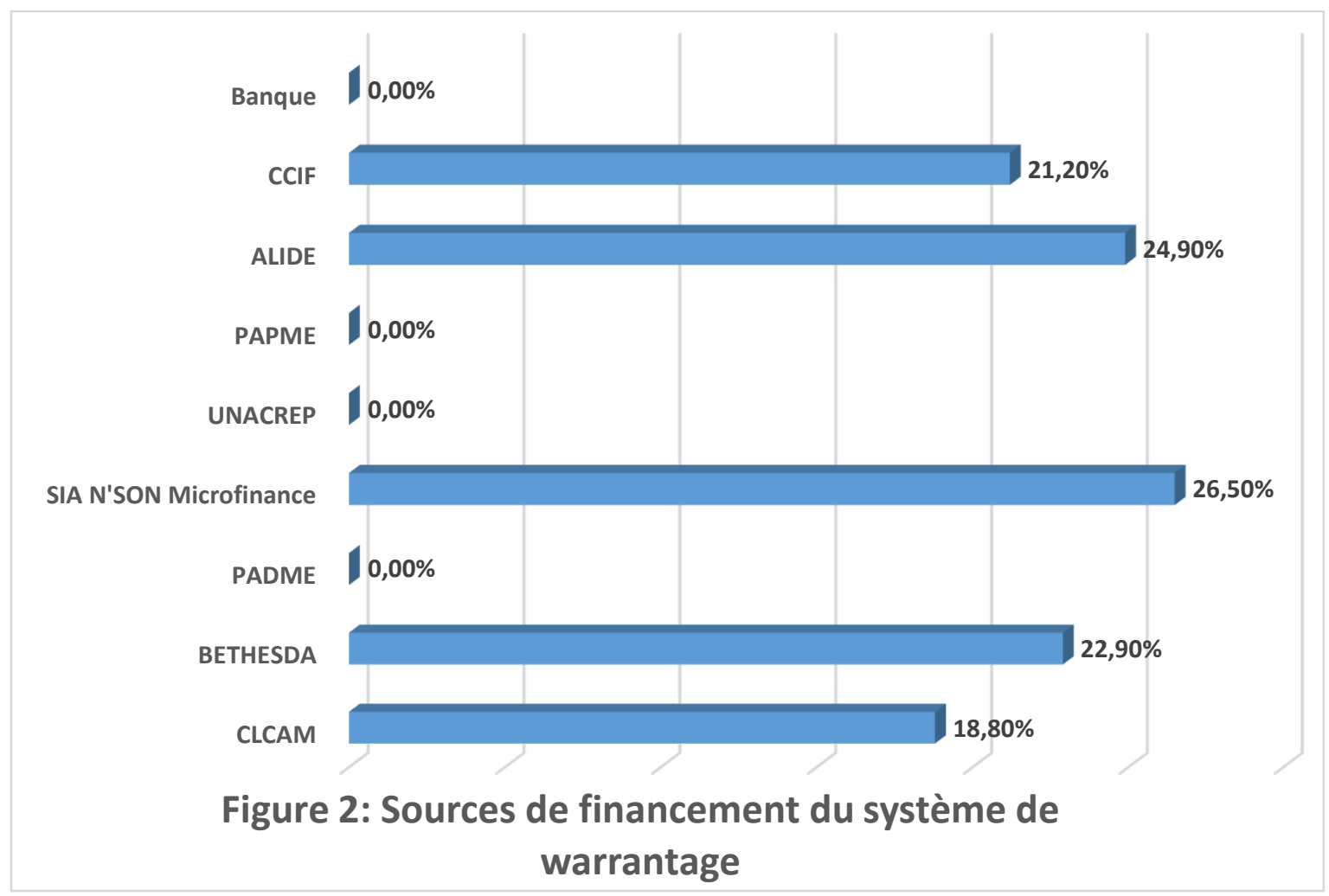

Source : Enquêtes 2019

Légende :

$\mathrm{CCIF}=$ Coopérative Communale d'Intermédiation Financière

ALIDE $=$ Association de Lutte pour la promotion des Initiatives de Développement

PAPME $=$ Promotion de l'Appui aux Petites et Moyennes Entreprises

UNACREP= Union Nationale des Caisses Rurales d'Epargne et de Prêt

PADME $=$ Promotion de 'Appui au Développement des Micro - Entreprises

CLCAM= Caisse Locale de Crédit Agricole Mutuel

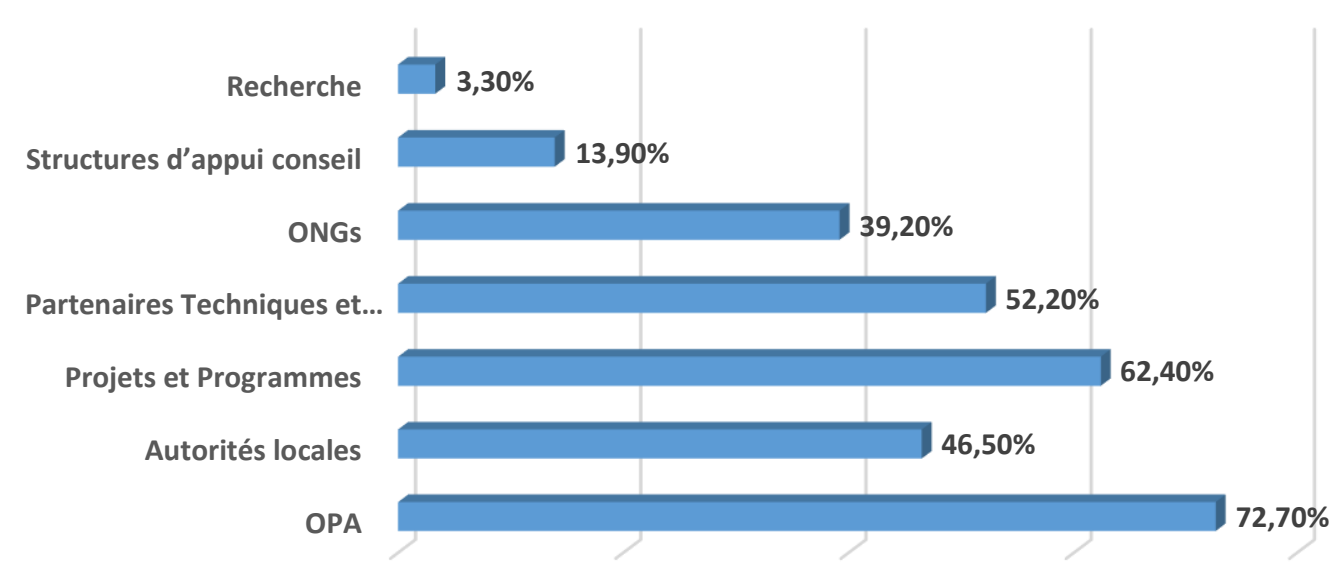

Figure 3: Principaux partenaires impliqués

Légende :

ONGs Organisations Non Gouvernementales

OPA=Organisation Professionnelles Agricoles. 
Tableau 2 : Affectations et stratégies de remboursement de crédit-warrantage.

\begin{tabular}{lc}
\hline Paramètres & Fréquence (\%) \\
\hline AFFECTATIONS DU CREDIT-WARRANTAGE & 55 \\
Développement d'AGR & 28 \\
Commercialisation & 12 \\
Paiement de la main-d'œuvre & 9 \\
Achat des intrants vivriers & 5 \\
Elevage / embouche & 1 \\
Transformation de produits agricoles & 45 \\
Charges sociales & 32 \\
Construction de maison d'habitation & 5 \\
Scolarisation des enfants & 4 \\
Consommation du ménage & 2 \\
Dépenses de santé & 2 \\
Diverses cérémonies & STRATÉGIES DE REMBOURSEMENT DU CREDIT-WARRANTAGE \\
& 42 \\
Activités de commercialisation développée & 33 \\
Vente des produits stockés & 20 \\
Activités de production agricole & 4 \\
Elevage / embouche & 1 \\
Transformation des produits agricoles & 1 \\
Autres activités &
\end{tabular}

Tableau 3 : Principaux éléments de coûts et recettes associés au stockage-warrantage.

\begin{tabular}{|c|c|c|c|c|}
\hline \multirow[t]{2}{*}{ Paramètres } & \multicolumn{2}{|c|}{ Mais } & \multicolumn{2}{|c|}{ Soja } \\
\hline & Moyenne & Ecart-type & Moyenne & Ecart-type \\
\hline Quantité de produits (maïs) stockés & 19164,01 & 30462,98 & 1711,75 & 2973,037 \\
\hline $\begin{array}{l}\text { Prix de vente du produit au moment de } \\
\text { la mise en stock }\end{array}$ & 107,105 & 26,321 & 110,425 & 39,019 \\
\hline $\begin{array}{l}\text { Montant du crédit-warrantage reçu en } \\
\text { contrepartie }\end{array}$ & 372000 & 690000 & 124000 & 169000 \\
\hline $\begin{array}{l}\text { Valeur de l'épargne nantie pour obtenir } \\
\text { le crédit-warrantage }\end{array}$ & 68552,33 & 228000 & 65745,63 & 279000 \\
\hline Valeur des frais de dossiers payés & 1446,819 & 2799,447 & 122,875 & 294,81 \\
\hline Valeurs des frais de gestion payés & 3551,214 & 15403,95 & 142,5 & 324,936 \\
\hline $\begin{array}{l}\text { Durée du stockage des produits (en } \\
\text { mois) }\end{array}$ & 5,097 & 1,511 & 4,825 & 1,852 \\
\hline $\begin{array}{l}\text { Quantité totale du produit stocké } \\
\text { prélevée pour la consommation }\end{array}$ & 0 & 0 & 39,8 & 169,138 \\
\hline Quantité totale du produit stocké vendu & 19164,01 & 30462,98 & 1671,95 & 2990,35 \\
\hline $\begin{array}{l}\text { Moment de la vente (nombre de mois } \\
\text { après) }\end{array}$ & 4,059 & 2,287 & 4,8 & 2,003 \\
\hline Prix de revente & 135,979 & 47,594 & 157,525 & 58,564 \\
\hline Montant total remboursé & 400000 & 743000 & 128000 & 183000 \\
\hline $\begin{array}{l}\text { Taux d'intérêt moyen payé sur le crédit- } \\
\text { warrantage }\end{array}$ & 6,82 & 2,489 & 4,4 & 2,968 \\
\hline
\end{tabular}


F. T. OGOUVIDE et al. / Int. J. Biol. Chem. Sci. 15(5): 2008-2022, 2021

Tableau 4 : Comparaison des moyennes des marges nettes des modèles de warrantage.

\begin{tabular}{|c|c|c|c|c|c|c|c|c|}
\hline \multirow{2}{*}{$\begin{array}{l}\text { Système de } \\
\text { warrantage }\end{array}$} & \multirow{2}{*}{$\begin{array}{l}\text { Système de } \\
\text { warrantage }\end{array}$} & \multirow[t]{2}{*}{ Moyenne } & \multirow[t]{2}{*}{ Ecart-Type } & \multirow{2}{*}{$\begin{array}{c}\text { Différence } \\
\text { moyenne (I-J) }\end{array}$} & \multirow{2}{*}{$\begin{array}{c}\text { Erreur } \\
\text { standard }\end{array}$} & \multirow[t]{2}{*}{ Sig. } & \multicolumn{2}{|c|}{ Intervalle de confiance à $95 \%$} \\
\hline & & & & & & & $\begin{array}{c}\text { Borne } \\
\text { inférieure }\end{array}$ & $\begin{array}{c}\text { Borne } \\
\text { supérieure }\end{array}$ \\
\hline \multirow[t]{3}{*}{ Accès-intrants } & AGR & 1369324,69 & 5266815,17 & $-1165353,69$ & 4820832,03 & 0,810 & $-10735915,32$ & 8405207,94 \\
\hline & Commercialisation & 289889,72 & 90117,447 & 875463,97 & 5051622,93 & 0,986 & $-10114658,21$ & 9942820,76 \\
\hline & Satisfaction-besoins & 306954,75 & 204093,78 & $-102983,75$ & 4240005,29 & 0,501 & $-11283051,29$ & 5551898,24 \\
\hline \multirow[t]{3}{*}{ AGR } & Accès-intrants & 203971,00 & 67599,264 & 1165353,69 & 4820832,03 & 0,810 & $-8405207,94$ & 10735915,32 \\
\hline & Commercialisation & 289889,72 & 90117,447 & 1079434,97 & 4652471,45 & 0,817 & $-8156888,65$ & 10315758,59 \\
\hline & Satisfaction-besoins & 306954,75 & 204093,78 & 1062369,94 & 3755561,38 & 0,652 & $-9155954,84$ & 5755509,17 \\
\hline \multirow[t]{3}{*}{ Commercialisation } & Accès-intrants & 203971,00 & 67599,264 & 85918,72 & 5051622,93 & 0,986 & $-9942820,76$ & 10114658,21 \\
\hline & AGR & 1369324,69 & 526681,517 & $-1079434,97$ & 4652471,45 & 0,817 & $-10315758,59$ & 8156888,65 \\
\hline & Satisfaction-besoins & 306954,75 & 204093,78 & $-17065,03$ & 4047556,55 & 0,494 & $-10815073,50$ & 5255757,90 \\
\hline \multirow[t]{3}{*}{ Satisfaction-besoins } & Accès-intrants & 203971,00 & 67599,264 & 102983,75 & 4240005,29 & 0,501 & $-5551898,24$ & 11283051,29 \\
\hline & AGR & 1369324,69 & 526681,517 & $-1062369,94$ & 3755561,38 & 0,652 & $-5755509,17$ & 9155954,84 \\
\hline & Commercialisation & 289889,72 & 90117,447 & 17065,03 & 4047556,55 & 0,494 & $-5255757,90$ & 10815073,50 \\
\hline
\end{tabular}

*** Significatif au seuil de 1\% ; ** Significatif au seuil de 5\% ; * Significatif au seuil de 10\%

Sources : Enquêtes 2019 
F. T. OGOUVIDE et al. / Int. J. Biol. Chem. Sci. 15(5): 2008-2022, 2021

Tableau 6 : Comparaison des moyennes des taux de rentabilité interne des modèles de warrantage.

\begin{tabular}{|c|c|c|c|c|c|c|c|c|}
\hline \multirow[t]{2}{*}{$\begin{array}{l}\text { Système de } \\
\text { warrantage }\end{array}$} & \multirow[t]{2}{*}{$\begin{array}{l}\text { Système de } \\
\text { warrantage }\end{array}$} & \multirow[t]{2}{*}{ Moyenne } & \multirow[t]{2}{*}{$\begin{array}{l}\text { Ecart- } \\
\text { Type }\end{array}$} & \multirow[t]{2}{*}{$\begin{array}{c}\text { Différence } \\
\text { moyenne (I-J) }\end{array}$} & \multirow[t]{2}{*}{ Erreur standard } & \multirow[t]{2}{*}{ Sig. } & \multicolumn{2}{|c|}{$\begin{array}{c}\text { Intervalle de confiance à } 95 \\
\%\end{array}$} \\
\hline & & & & & & & $\begin{array}{c}\text { Borne } \\
\text { inférieure }\end{array}$ & $\begin{array}{c}\text { Borne } \\
\text { supérieure }\end{array}$ \\
\hline \multirow[t]{3}{*}{ Accès-intrants } & AGR & 0,93 & 0,05 & $-0,041 * * *$ & 0,051 & 0,001 & $-0,143$ & 0,061 \\
\hline & Commercialisation & 0,94 & 0,05 & $-0,045 * * *$ & 0,054 & 0,001 & $-0,152$ & 0,062 \\
\hline & Satisfaction-besoins & 0,75 & 0,18 & $0,147 * * *$ & 0,045 & 0,002 & 0,058 & 0,237 \\
\hline \multirow[t]{3}{*}{ AGR } & Accès-intrants & 0,89 & 0,22 & $0,041 * * *$ & 0,051 & 0,001 & $-0,061$ & 0,143 \\
\hline & Commercialisation & 0,94 & 0,05 & $-0,004$ & 0,050 & 0,930 & $-0,103$ & 0,094 \\
\hline & Satisfaction-besoins & 0,75 & 0,18 & $0,188 * * *$ & 0,040 & 0,000 & 0,109 & 0,267 \\
\hline \multirow[t]{2}{*}{ Commercialisation } & Accès-intrants & 0,89 & 0,22 & $0,045^{* * *}$ & 0,054 & 0,001 & $-0,062$ & 0,152 \\
\hline & AGR & 0,93 & 0,05 & 0,004 & 0,050 & 0,930 & $-0,094$ & 0,103 \\
\hline \multirow[t]{3}{*}{ Satisfaction-besoins } & Accès-intrants & 0,89 & 0,22 & $-0,147 * * *$ & 0,045 & 0,002 & $-0,237$ & $-0,058$ \\
\hline & AGR & 0,93 & 0,05 & $-0,188 * * *$ & 0,040 & 0,000 & $-0,267$ & $-0,109$ \\
\hline & Commercialisation & 0,94 & 0,05 & $-0,192 * * *$ & 0,043 & 0,000 & $-0,278$ & $-0,107$ \\
\hline
\end{tabular}

*** Significatif au seuil de $1 \%$;** Significatif au seuil de $5 \%$; * Significatif au seuil de $10 \%$

Sources : Enquêtes 2019. 


\section{DISCUSSION}

Les quatre modèles de warrantage identifiés par Egah et al. (2015) ont été rencontrées. Le plus dominant est le warrantage-puriel, rencontré chez $43 \%$ des producteurs. Cette prédominance du warrantage-pluriel pourrait être expliquée par le fait que, après avoir utilisé les ressources disponibles pour les charges de production, les producteurs ont des besoins monétaires importants pour non seulement faire face au remboursement des dettes enregistrées (intrant, main-d'œuvre, ...) et aux charges sociales (scolarité des enfants, cérémonies...), mais aussi pour s'acheter quelques intrants en réserve pour la campagne à venir (Augères, 2007). Ce modèle de warrantage est suivi du warrantage-AGR, rencontré chez $29 \%$ des producteurs impliqués. Ensuite est venu le warrantage-commercialisation, rencontré chez $18 \%$ des producteurs enquêtés. Le warrantageintrant, rencontré chez $10 \%$ des producteurs enquêtés, est le moins pratiqué.

Quel que soit le modèle de warrantage, les acteurs impliqués sont diversifiés comme rapporté par les études antérieures (Sogodogo et al., 2014 ; Garrido et Sanchez, 2015 ; Egah, 2016).

Les marges nettes d'exploitation obtenues pour les quatre modèles de warrantage sont positives. Cette rentabilité du système de warrantage au Bénin a été confirmée par Faladé (2011) qui a rapporté que «l'expérience du warrantage a généré aux ménages un gain de 29,4 FCFA/ kg de maïs et un gain supplémentaire de plus de 17 milliards FCFA en 2010 pour l'ensemble des producteurs du maïs du Bénin ». Cependant, ce résultat infirme ceux de Garrido et Sánchez (2015) qui ont montré que « la participation des producteurs au warrantage au Burkina Faso a généré des bénéfices plus faibles (voire des pertes) que les gains qui auraient pu être obtenus en vendant les céréales après la récolte ».
Cependant, l'analyse par modèle indique que le warrantage-AGR semble être économiquement plus rentable, suivi du warrantage-commercialisation, du warrantagePluriel et enfin du warrantage-intrant. Cette meilleure rentabilité du warrantage-AGR s'explique par les revenus additionnels générés par les activités économiques développées avec le crédit obtenu (Augères, 2007). Le développement d'AGR en association au warrantage permet aussi de faire face au risque de non-augmentation suffisante des prix des produits stockés et de l'exigence de rembourser parfois le crédit avant l'ouverture de l'entrepôt, le stock servant de garantie du prêt (Garrido et Sanchez, 2015).

Concernant le TRI, les valeurs obtenues (75\% à 94\%) ont montré que les quatre systèmes de warrantage permettent tous de rentabiliser le capital investi. Ce résultat confirme ceux de Ouattara et al. (2015) qui ont rapporté que «le système de warrantage a permis aux exploitants du Burkina Faso de générer des profits de l'ordre de 90 à $183 \%$ et a une influence significative sur la décision d'adopter une technique de microdosage ». De même, il confirme ceux de Coulter et Mahamadou (2009), qui ont affirmé que «le warrantage a permis aux paysans nigériens de générer en six mois, un gain important $(19 \%$ à $113 \%$ ) sur leur capital investi et d'adopter les technologies (microdose) pour améliorer le rendement ».

Par ailleurs, les valeurs moyennes des Taux de Rentabilité Interne (TRI) obtenues ont montré que le warrantage-commercialisation et le warrantage-AGR étaient plus rentables en termes du capital investi. Ils étaient suivis, respectivement, du warrantage-intrant et du warrantage pluriel. Cette meilleure rentabilité du warrantage-commercialisation particulièrement, par rapport au capital investi, s'explique par la proximité des pratiquants de ce modèle des principales villes du Sud-Bénin (Cotonou, Porto-Novo) et du Nigéria, où les prix des produits agricoles sont meilleurs. De 
même, la vente groupée qui caractérise ce modèle de warrantage, de par le volume de produits mis en vente, permet aux organisations de producteurs d'être en position de force pour négocier et obtenir des conditions plus favorables de vente (Sogodogo et al., 2014 ; Garrido et Sanchez, 2015).

\section{Conclusion}

Le warrantage mis en œuvre au Bénin est assez rentable et varie suivant le modèle pratiqué. Cependant en considérant la marge nette, le modèle de warrantage-AGR semble être économiquement plus rentable, suivi du warrantage-commercialisation, du warrantagepluriel et enfin du warrantage-intrant. Par contre, le warrantage-commercialisation et le warrantage- AGR sont plus rentables, en termes de capital investi. Ils sont suivis du warrantage-intrant et du warrantage pluriel. Ces résultats militent en faveur de la promotion des systèmes de warrantage basés sur la commercialisation et le développement des activités génératrices de revenus (AGR). Par ailleurs, pour permettre d'améliorer d'avantage la rentabilité du système de warrantage au Bénin, nous suggérons de :

(i) réduire du taux d'intérêt appliqué sur le crédit-warrantage ;

(ii) retarder la mise en vente des produits warrantés, pour permettre aux prix du marché d'augmenter d'avantage ;

(iii) mettre en place un système d'information sur les prix des marchés (SIM) des produits agricoles, au profit des producteurs impliqués dans le système de warrantage au Bénin.

\section{CONFLITS D'INTERETS}

Les auteurs déclarent ne pas avoir de conflits d'intérêts concurrents.

\section{CONTRIBUTIONS DES AUTEURS}

Ce travail a été réalisé en collaboration entre tous les trois auteurs. FTO et LCH ont conçu l'étude, conduit l'enquête exploratoire, conçu le questionnaire d'enquête via une plateforme ODK Kobocollect. FTO, LCH et RNAA ont supervisé la collecte des données sur le terrain. FTO a effectué les analyses statistiques et élaborer le premier draft du manuscrit, avec l'appui de LCH. RNAA a supervisé l'ensemble du travail et examiné toutes les ébauches du manuscrit. Tous les auteurs ont lu et approuvé le manuscrit final.

\section{REMERCIEMENTS}

Les auteurs remercient le Programme d'Amélioration de la Productivité Agricole des Petits Exploitants (PAPAPE) financé par la Banque Islamique de Développement (BID), pour le soutien financier de l'étude. Ils remercient également les informateurs clés que constituent les acteurs impliqués dans le warrantage des produits vivriers, pour leur disponibilité et les informations fournies.

\section{REFERENCES}

Abdoulaye T, Sanders JH. 2003. Improving Marketing Strategies to Accelerate Technological change+ for the basic Cereal: The Niger Case. American Agricultural Economics Association Annual Meeting, July 27-30, 2003. Montreal, Canada 22207, 18p. DOI: 10.22004/ag.econ.22207

Augères CB.2007. Le warrantage ou crédit stockage : un moyen pour les paysans de mieux valoriser leurs produits et une sécurisation du financement rural. Communication au Forum régional Bamako sur le thème "Valorisation d'expériences d'organisations paysannes: «Accès au marché et commercialisation de produits agricoles»". 16-18 janvier 2007, Inter-réseaux Développement rural - CTA. $17 \mathrm{p}$

Ba R, Monteiro NMF, Hounguè U, Donou Hounsodé MT, Gbaguidi F, BabaMoussa L. 2016. Perception des producteurs et impact des facteurs socioéconomiques sur la connaissance des 
mycotoxines du maïs en stockage au Bénin. Int. J. Biol. Chem. Sci., 10(1): 155166.

DOI:

http://dx.doi.org/10.4314/ijbcs.v10i1.11

Barthélemy O. 2013. La microfinance et les difficultés de financement du monde rural : l'exemple des impayés au sein de la MECFEPRODES. Mémoire de fin d'étude, Sciences Po Toulouse Master « Développement économique et coopération internationale $», 107 \mathrm{p}$

Coulter J, Mahamadou S. 2009. Revue du warrantage paysan au Niger. Version définitive, Agence Française de Développement (AFD), 61p.

Daley-Harris S. 2009. State of the Microcredit Summit Campaign Report. https://core.ac.uk/download/pdf/1117716 1.pdf.

Duffau A, Lagandré D, Chetaille A, Rozenkopf I, Horréard G. 2011. Assurance indicielle et warrantage, quel intérêt pour les petits agriculteurs? Coll. Études et travaux (28), Editions du Gret, 44p.

Egah J. 2016. Analyse des formes de warrantage autour du maïs et leurs incidences sur la sécurité alimentaire des ménages ruraux du Bénin. Thèse de Doctorat unique en Socio-Economie du Développement, Faculté des Sciences Agronomiques (FSA), Université d'Abomey-Calavi, Bénin, 244 p.

Egah J, Baco MN, Yegbemey RN, Tossou CR. 2015. Analysis of Organizational Innovations Adoption: cases of the Different forms of Maize-related Warrantage in Benin. Journal of Education, Society and Behavioural Science, $\quad$ 6(4): $227-240$. DOI: 10.9734/BJESBS/2015/15447

Egah J, Baco MN, Moumouni MI, Akponikpè PBI, Yegbemey RN, Tossou CR. 2014. Performance of Institutional Innovation: The Case of Maize-Related Warrantage in Benin, West Africa. International
Journal of Agriculture Innovations and Research, 3(2): 8 p.

Faladé D. 2011. Le warrantage (stockagecrédit) : facteurs déterminants, succès et échecs. Rapport d'étude réalisée pour le compte de la Fédération des Unions de Producteurs du Bénin (FUPRO-Bénin). Septembre 20111, $94 \mathrm{p}$.

Fall M, Lanha M. 2013. Antisélection et Institutions de Microfinance. Communication aux Vèmes Journées Internationales de la Microfinance, Douala, 11, 12 et 13 Septembre 2013 sur le thème : "Mutations et Crises de la microfinance $», 15 \mathrm{p}$

Garrido ES, Sánchez IS. 2015. Warrantage paysan au Burkina Faso : Accès au crédit par le biais des stocks de proximité. Rapports de recherche OXFAM, Octobre 2015, $61 \mathrm{p}$

Guirkinger, C, Boucher SR. 2008. Credit constraints and productivity in Peruvian agriculture. Agricultural Economics, 39(3): 295-308. DOI: 10.1111/j.15740862.2008.00334.x

Houédjissin R, Allagbé MC, Kodjo MK. 2002. Diagnostic des institutions de microfinance au sud et au centre du Bénin. In Agbo, B. P. et al., 2002. Actes Scientifiques 2 de la recherche Agricole au Bénin.

Manirakiza D, Ndimanya P, Lebailly P. 2018. Les effets du warrantage sur la croissance des organisations paysannes au Burundi : cas des coopératives agricoles de la Confédération des Associations de Producteurs Agricoles pour le Développement (CAPAD). Les Cahiers de l'Association Tiers-Monde n³3-2018.

Ouattara B, Taonda SJB, Traoré A, Lompo F, Sédogo PM, Baco MN, Bationo A, Koala S, Kimaro A, Peak D. 2015. Use of an inventory credit system called warrantage to combat rural poverty and hunger in the semi-arid area of Burkina Faso. 13 p. 
Paraïso A, Yabi AJ, Sossou A, ZoumarouWallis N, Yegbemey R. 2012. Rentabilité Economique et Financière de la Production Cotonnière à Ouaké au NordOuest du Bénin. Annales des Sciences Agronomiques, 16 (1) : 91-105.

Sogodogo D, Dembele O, Konate S, Koumare S. 2014. Contribution du warrantage a l'accès des petits producteurs au marché des intrants et des produits agricoles dans les communes rurales de Klela, Fama et Zebala dans la région de Sikasso au Mali. Agronomie Africaine, 26 (2) : 167 - 179.

Tabo R, Bationo A, Hassane A, Amadou B, Fosu M, Sawadogo-Kabore S. Fatondji D, Korodjouma O, Abdou A, Koala S. 2008. Fertilizer microdosing for the prosperity of resource poor farmers: A success story. Proceedings of the Workshop on Increasing the Productivity and Sustainability of Rainfed Cropping Systems of Poor, Smallholder Farmers, Tamale, Ghana, 22-25 September 2008. Report pp 269-277.
Yabi AJ. 2009. Efficiency in rice production: Evidence from Gogounou District in the North of Benin. Annales des Sciences Agronomiques, 12 (2): 61-75.

Yabi AJ, Paraïso A, Ayena RL, Yegbemey R. 2012. Rentabilité économique de production agricole sous pratiques culturales de gestion de la fertilité des sols dans la commune de Ouaké au nord-ouest $\mathrm{du}$ Bénin. Annales des Sciences Agronomiques, 16 (2) : 229-242.

Yabi JA, Bachabi FX, Labiyi IA, Ode CA, Ayena RL. 2016. Déterminants socioéconomiques de l'adoption des pratiques culturales de gestion de la fertilité des sols utilisées dans la commune de Ouaké au NordOuest du Bénin. Int. J. Biol. Chem. Sci., 10(2): 779-792. DOI: http://dx.doi.org/10.4314/ijbcs.v10i2.27

Yegbemey RN. 2010. Analyse économique des exploitations rizicoles de la Commune de Malanville. Thèse d'ingénieur agronome, Faculté d'Agronomie, Université de Parakou, Bénin ; 75 p. 